\title{
Gastropleural fistula due to gastric lymphoma presenting as tension pneumothorax and empyema
}

\author{
C.J. Warburton, P.M.A. Calverley
}

Gastropleural fistula due to gastric lymphoma presenting as tension pneumothorax and empyema. C.J. Warburton, P.M.A. Calverley. (CERS Journals Ltd 1997.

ABSTRACT: The formation of a gastropleural fistula is an uncommon complication of a number of conditions.

The case of a patient with gastropleural fistula, as a complication of gastric lymphoma, is presented. The patient developed a tension pneumothorax and empyema. On cytological examination of the empyematous fluid, the presence of food debris aided diagnosis.

The patient died 2 days after a total gastrectomy. The development of this complication in gastric lymphoma appears to have a particularly poor prognosis. Eur Respir J 1997; 10: 1678-1679.

\author{
Aintree Chest Centre, Fazakerley Hospital, \\ Liverpool, UK \\ Correspondence: C.J. Warburton \\ Aintree Chest Centre \\ Fazakerley Hospital \\ Liverpool \\ UK \\ Keywords: Gastropleural fistula \\ lymphoma \\ Received: October 311996
}

Accepted after revision March 311997
Gastropleural fistula is an uncommon complication of a number of disease processes. We describe a case of gastropleural fistula that presented as a tension pneumothorax and empyema as a result of a gastric lymphoma. An enteropleural fistula was confirmed by cytology of the empyematous fluid, and also radiologically with a gastrograffin swallow.

\section{Case history}

A 65 yr old female was referred for a gastroenterological opinion from her general practitioner, with a recrudescence of symptoms of epigastric pain, anorexia and nausea. She had been investigated for similar symptoms 7 yrs previously, when she was found to have chronic gastric ulceration. She underwent multiple upper gastrointestinal (GI) endoscopies, as some of the gastric biopsies were thought to be suspicious of lymphoma. Despite review by the British National Lymphoma Investigation panel, no firm diagnosis of malignancy could be made, and her symptoms (and gastric ulceration) disappeared entirely with omeprazole therapy. Helicobacter pylori was never detected. The patient was observed with repeat endoscopies and biopsies for 4 yrs, during which time she remained well, and was subsequently discharged from follow-up 3 yrs prior to her rereferral.

Upper GI endoscopy at the time of rereferral demonstrated multiple polypoidal lesions in the gastric cardia. Biopsies from these lesions showed a B-cell malignant lymphocytic non-Hodgkins lymphoma of the mucosa-associated lymphoid tissue (MALT). There was no evidence of enlarged peripheral lymphadenopathy, and computed tomography (CT) scanning of the thorax, abdomen and pelvis, and a blood film and bone marrow examination were normal. The patient was prescribed oral chemotherapy in the form of chlorambucil, $10 \mathrm{mg}$ daily. Two months after commencing this therapy, she had a repeat endoscopy, and this time a biopsy showed transformation to a high grade immunoblastic non-Hodgkins lymphoma. She was admitted for assessment for total gastrectomy.
Two days following admission. the patient complained of acute, severe, left-sided pleuritic chest pain, associated with shortness of breath. There was no objective evidence of pulmonary embolism, with normal arterial blood gas values and no electrocardiographic changes. A chest radiograph was reported as showing some left basal pneumonic shadowing only, and she was treated with antibiotics. Her condition deteriorated further the same day, she became cold, clammy, tachypnoeic and tachycardic. Her trachea was noted to be deviated to the right and her left hemithorax was hyperresonant, with absent breath sounds. A repeat chest radiograph confirmed a tension hydropneumothorax (fig. 1), and an intercostal chest drain was inserted without delay, with good effect.

A respiratory opinion was requested the following day, by which time the patient was peripherally shut-down and hypotensive. There was dark brown fluid draining from her left hemithorax, which failed to turn blue litmus paper to red, and which on Gram staining showed leucocytes and Gram-positive bacilli. An enteropleural

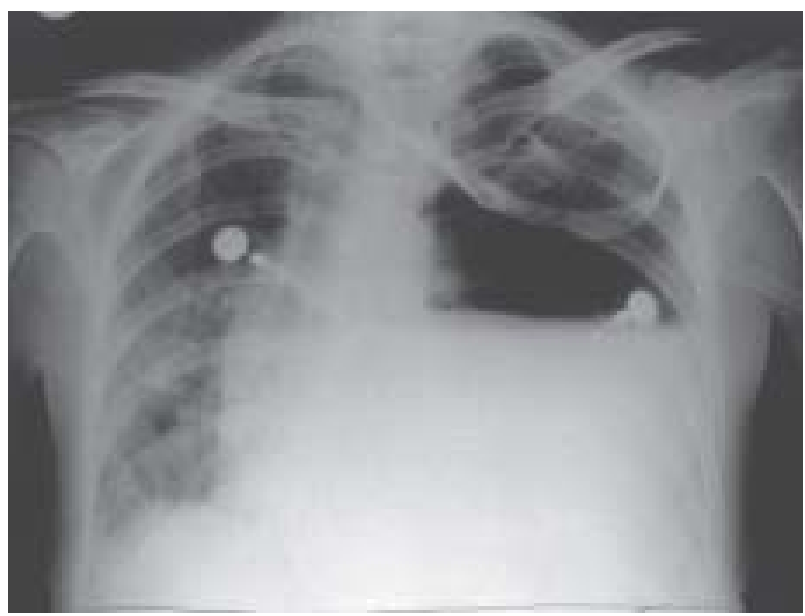

Fig. 1. - Chest radiograph showing tension hydropneumothorax. 


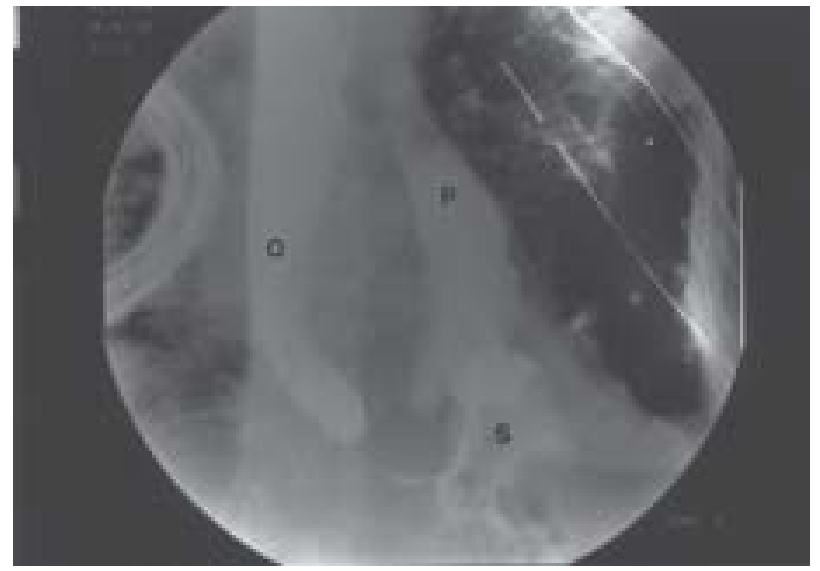

Fig. 2. - Gastrograffin swallow demonstrating contrast in the oesophagus (O), an abnormal stomach (S) and flow of contrast into the left pleural cavity $(\mathrm{P})$.

fistula was suspected, and this was confirmed by cytology of the empyematous fluid, which revealed food debris. A gastrograffin swallow was contemplated but had to be delayed until drainage of the radiodense pleural fluid had occurred. When it was performed, leakage of contrast from the stomach into the left pleural space was demonstrated when the patient was supine (fig. 2). The initial chest radiograph was reviewed, and this, in fact, had shown a small apical left pneumothorax along with the reported left basal pneumonic shadowing.

During the subsequent $48 \mathrm{~h}$, her condition continued to deteriorate both clinically and biochemically. Urgent staging investigations were performed, which revealed a 2-3 cm mass of para-aortic lymph nodes but no other abnormality on CT scanning or bone marrow examination. In view of her deterioration and the relatively localized nature of her disease, it was decided that a total gastrectomy was the only option, in spite of her abnormal biochemistry (sodium $128 \mathrm{mmol} \cdot \mathrm{L}^{-1}$, potassium 3.2 $\mathrm{mmol} \cdot \mathrm{L}^{-1}$, albumin $18 \mathrm{~g} \cdot \mathrm{L}^{-1}$ ), mild anaemia (haemoglobin $10.8 \mathrm{~g} \cdot \mathrm{dL}^{-1}$ ), abnormal clotting studies (international normalized ratio (INR) 2.3, activated partial thromboplastin time (APTT) ratio 2.2), and presumed septicaemia.

The operation confirmed a fistula between the fundus of the stomach and the left pleural cavity through the left hemidiaphragm. A total gastrectomy and splenectomy was carried out; however, the patient died 2 days postoperatively, despite intensive therapy.

\section{Discussion}

Pneumothorax developing as a result of oesophageal rupture has been well-described, especially as a result of instrumentation [1], although spontaneous rupture also occurs [2]. Perforation of the stomach and colon have also been described, causing pneumothorax when this occurs in conjunction with congenital diaphragmatic hernias $[3,4]$. Fistulation between the stomach and the pleural cavity through the diaphragm is rare, but has been described as a result of peptic ulceration [5], surgery [6, 7], trauma [7], and gastric malignancy. Gastric lymphomas account for less than $5 \%$ of all gastric neoplasms [8], and the prognosis of the tumour is dependent upon the stage of disease at presentation. The $5 \mathrm{yr}$ survival for gastric lymphoma varies overall from approximately 47 to $57 \%$ [8]. Although perforation is said to occur in up to $14 \%$ of gastric lymphomas [7], only two previous reports of gastropleural fistula as a complication of gastric lymphoma have been described. Neither of these cases presented with the combination of empyema and tension pneumothorax seen in our patient [7, 9].

The diagnosis of gastropleural fistula is usually made with contrast radiology, upper GI endoscopy, or at operation. The use of methylene blue as a marker, and the testing of the pleural fluid for $\mathrm{pH}$ or bile salts have been suggested as easy bedside tests for the presence of a gastropleural fistula in ill patients [7]. The $\mathrm{pH}$ of our patient's pleural fluid was not helpful, and this was probably related to her omeprazole therapy, which largely suppresses gastric acid secretion. In this patient, the finding of food debris within the fluid was indicative of an enteropleural fistula, although the exact site of fistula was obviously not identified. We are not aware of any previous descriptions of the use of cytology in this context.

The lymphoma had probably been present in our patient for at least 7 yrs. It is unclear why the fistula formed after such a long time, but it may be related to the transformation of the tumour to a higher grade of malignancy, or possibly due, in part, to the institution of cytotoxic chemotherapy.

The prognosis of fistulas from the upper GI tract to the pleura seems to depend upon the delay from diagnosis to surgical intervention [10, 11]. It is important, therefore, to consider this diagnosis early in the patient's management. The development of this rare complication in gastric lymphoma appears to represent a particularly poor prognostic sign, with death resulting in two of the three cases described (including the present patient) within days, usually due to postoperative sepsis.

\section{References}

1. Ghahremani GG, Turner MA, Port RB. Iatrogenic intubation injuries to the upper gastrointestinal tract in adults. Gastrointest Radiol 1980; 5: 1-10.

2. Naylor AR, Walker WS, Dark J, Cameron EWJ. T-tube intubation in the management of seriously ill patients with oesophagopleural fistula. Br J Surg 1990; 77: 40-42.

3. Nayak LN, Lawrence D. Tension pneumothorax from a perforated gastric ulcer. Br J Surg 1976; 63: 245-247.

4. Price BA, Elliott MJ, Featherstone G, Blesovsky A. Perforation of intrathoracic colon causing acute pneumothorax. Thorax 1983; 38: 959-960.

5. Roberts CM, Gelder CM, Goldstraw P, Spiro SG. Tension pneumothorax and empyema as a consequence of gastropleural fistula. Respir Med 1990; 84: 53-54.

6. O'Keefe PA, Goldstraw P. Gastropleural fistula following pulmonary resection. Thorax 1993; 48: 1278-1279.

7. Rotstein OD, Pruett TL, Simmons RC. Gastropleural fistula: report of three cases and review of literature. Am J Surg 1985; 150: 392-396.

8. Davis GR. Neoplasms of the stomach. In: Sleisenger MH, Fordtran JS, eds. Gastrointestinal Disease. 4th Edn. London, W.B. Saunders, 1989; pp. 761-763.

9. Cobo J, Gomez-Cerezo J, Molina F, Medrano JC. Gastropleural fistula: primary manifestation of gastric lymphoma (Letter). Med Clin (Barc) 1990; 94(19): 757.

10. Nesbitt JC. Surgical management of perforation of the oesophagus. Am J Surg 1987; 53: 183-191.

11. Bladergoen MR. Diagnosis and management of oesophageal perforation and rupture. Ann Thorac Surg 1986; 42: 235-239. 